\title{
OBSERVER-BASED FUZZY COVARIANCE CONTROL FOR DISCRETE NONLINEAR SYSTEMS
}

\author{
Wen-Jer Chang \\ Professor, Department of Marine Engineering, National Taiwan Ocean University, Keelung Taiwan 202, R.O.C., \\ wjchang@mail.ntou.edu.tw \\ Yi-Lin Yeh \\ Master, Department of Marine Engineering, National Taiwan Ocean University, Keelung Taiwan 202, R.O.C.
}

Follow this and additional works at: https://jmstt.ntou.edu.tw/journal

Part of the Electrical and Computer Engineering Commons

\section{Recommended Citation}

Chang, Wen-Jer and Yeh, Yi-Lin (2007) "OBSERVER-BASED FUZZY COVARIANCE CONTROL FOR DISCRETE NONLINEAR SYSTEMS," Journal of Marine Science and Technology. Vol. 15: Iss. 3, Article 11.

DOI: 10.51400/2709-6998.2399

Available at: https://jmstt.ntou.edu.tw/journal/vol15/iss3/11

This Research Article is brought to you for free and open access by Journal of Marine Science and Technology. It has been accepted for inclusion in Journal of Marine Science and Technology by an authorized editor of Journal of Marine Science and Technology. 


\section{OBSERVER-BASED FUZZY COVARIANCE CONTROL FOR DISCRETE NONLINEAR SYSTEMS}

\section{Acknowledgements}

The authors wish to express their sincere gratitude to three anonymous reviewers who gave them some constructive comments, criticisms and suggestions. This work was supported by the National Science Council of the Republic of China, under contract NSC93-2218-E019-002. 


\title{
OBSERVER-BASED FUZZY COVARIANCE CONTROL FOR DISCRETE NONLINEAR SYSTEMS
}

\author{
Wen-Jer Chang* and Yi-Lin Yeh**
}

Key words: takagi-sugeno fuzzy models, parallel distributed compensation, observed-state feedback gains and covariance control.

\section{ABSTRACT}

In this paper, the practical covariance control theory is applied to incorporate the concept of variance constrained control for the discrete nonlinear systems using T-S fuzzy models. This paper focuses on the control problem of finding observed-state feedback gains for the discrete fuzzy controllers, which can achieve the common state covariance assignment. Finally, a numerical example will be used to show the simulation results of the present fuzzy control method and usefulness of the proposed approach.

\section{INTRODUCTION}

This paper considers the discrete Takagi-Sugeno (T-S) fuzzy model $[9,24]$, that is described by a set of fuzzy "IF-THEN" rules with fuzzy sets in the antecedents and dynamics systems in the consequent. In this type of fuzzy model, local dynamics in different statespace regions are represented by linear models. Recently, its stability analysis and design problems have been considered in [25-27]. To solve this control problem, Tanaka and Wang proposed the design method of Parallel Distributed Compensation (PDC) [25-27] as a design framework. The goal of PDC method is to design linear feedback gain for each local linear model, and let the overall system input can be blended by these linear feedback gains. This method requires to find a common positive define matrix $\boldsymbol{P}$ such that the sufficient stability conditions are satisfied for every "IF-THEN" rule. Although looking for a common positive definite solution of the Lyapunov inequalities is by no means easy, the Lyapunov inequalities can be transformed into a set of

Paper Submitted 10/05/06, Accepted 12/08/06. Author for Correspondence: W.J.Chang. E-mail:wjchang@mail.ntou.edu.tw.

*Professor, Department of Marine Engineering, National Taiwan Ocean University, Keelung Taiwan 202, R.O.C.

**Master, Department of Marine Engineering, National Taiwan Ocean University, Keelung Taiwan 202, R.O.C.
Linear Matrix Inequalities (LMI) [3, 25, 26]. The LMIs can be solved by a numerical algorithm, which is an useful tool in finding a common positive definite matrix $\boldsymbol{P}$.

For the stochastic systems, many scholars have provided methodologies for designing the controllers by using the covariance control technology [4-6, 10-17, 23 ]. It has been proposed as an alternative approach for stochastic controller design. It is known that the quadratic optimization has been the most popular controller design method. However, it can guarantee only that the control system state vector as a whole behaves well. In order to deal with the individual variance constrained design problem, a methodology of designing controllers has been developed for several stochastic systems. The methodology is called as "Covariance Control Theory" [4-6, 10-17, 23]. In the covariance control theory, all state feedback gains are found which can assign the state covariance to a specified matrix value. This is extremely useful when it is desired to assign all the Root-Mean-Square (RMS) values of the individual states to specified values.

Over the past decade, a number of researchers have already given much insight into the problems related to the estimation and control theories of various dynamic systems. A series of results appeared in $[2,19-$ 22]. Most recently, the authors have already successfully dealt with the constrained variance design, based on the covariance control theory, for continuous nonlinear stochastic systems [8] and discrete nonlinear stochastic systems [7]. Nonetheless, [7, 8] assumed a priori that each of the system states can be measured accurately. Therefore, this work continues to discuss the nonlinear systems whose states are partially immeasurable. The main objective of this paper is to design the observed-state feedback laws which achieve the closed-loop system with a specified state covariance. Here, this problem is referred to as "Covariance Control with the Observed-State Feedback (CCOSF)". In this study, the state estimation theory and the covariance control theory will be combined to achieve individual performance objectives completely. The contribution 
of this approach is that the individual variance constraints can be achieved by combining covariance control theory and estimation theory for the nonlinear discrete stochastic systems whose states are partially immeasurable. In addition, the proposed approach provides another advantage that the covariance matrices of true states and estimation errors may be chosen separately to design observed-state feedback controllers and optimal filter gains.

The organization of this paper is presented as follows. Section 2 introduce the stability conditions of discrete T-S fuzzy control systems, then describes the control problem and discusses the optimal state estimation of discrete nonlinear stochastic systems using T-S fuzzy models. Section 3 will find the solutions of the CCOSF problem. In addition, the existence of observed-state feedback gains of T-S type fuzzy controllers will be developed and solved. In Section 4, a numerical simulation is presented to demonstrate the feasibility and applicability of this approach. Finally, conclusions are drawn in Section 5.

\section{DESCRIPTIONS OF DISCRETE OBSERVED- STATE FEEDBACK NONLINEAR SYSTEMS USING T-S FUZZY MODELS}

The fuzzy inference engine uses the fuzzy IFTHEN rules to perform a mapping from an input linguistic vector $x=\left[\begin{array}{llll}x_{1} x_{2} & \ldots & x_{n_{x}}\end{array}\right]^{T} \in \Re^{n_{x}}$ to an output variable $y \in \mathfrak{R}^{n_{y}}$. The system is described by fuzzy IF-THEN rules, which represent local linear input-output relations of nonlinear systems. In this section, a T-S type fuzzy stochastic model is used to construct a nonlinear stochastic system as follows.

\section{Plant Rule $i$ :}

IF $x_{1}(k)$ is $M_{i 1} \ldots$ and $x_{n_{x}}(k)$ is $M_{i n_{x}}$,

THEN $x(k+1)=\boldsymbol{A}_{i} x(k)+\boldsymbol{B}_{i} u(k)+\boldsymbol{D}_{i} v(k)$,

$$
y(k)=\boldsymbol{C}_{i} x(k)+\boldsymbol{E}_{i} \boldsymbol{\mu}(k), i=1,2, \ldots, r,
$$

where $x(k) \in \Re^{n_{x}}$ is the state vector; $u(k) \in \Re^{n_{u}}$ is the control input vector; and $y(k) \in \Re^{n_{y}}$ is the control output vector in i-th rule. The $v(k) \in \Re^{n_{v}}$ and $\mu(k) \in \Re^{n_{\mu}}$ are stationary zero-mean mutually independent white noise processes with covariance $\boldsymbol{V}>0$ and $\Omega>0$, respectively. The matrices, $\boldsymbol{A}_{i} \in \Re^{n_{x} \times n_{x}}, \boldsymbol{B}_{i} \in \Re^{n_{x \times} n_{u}}, \boldsymbol{C}_{i} \in \Re^{n_{y} \times n_{x}}$, $\boldsymbol{D}_{i} \in \Re^{n_{x} \times n_{v}}$, and $\boldsymbol{E}_{i} \in \Re^{n_{y} \times n_{\mu}}$ are constant; $i=1,2, \ldots$, $r$ and $r$ is the number of IF-THEN rules. The $M_{i j}$ are fuzzy sets and it is assumed that $\boldsymbol{B}_{i}$ is full-column rank. Besides, the pairs $\left(\boldsymbol{A}_{i}, \boldsymbol{B}_{i}\right)$ and $\left(\boldsymbol{A}_{i}, \boldsymbol{C}_{i}\right)$ are controllable and observable, respectively.

The state and output equations for the system can be represented in term of the rules (1) as

$$
\begin{aligned}
& x(k+1)=\sum_{i=1}^{r} h_{i}(k) \boldsymbol{A}_{i} x(k)+\sum_{i=1}^{r} h_{i}(k) \boldsymbol{B}_{i} u(k) \\
&+\sum_{i=1}^{r} h_{i}(k) \boldsymbol{D}_{i} v(k), \\
& y(k)=\sum_{i=1}^{r} h_{i}(k) \boldsymbol{C}_{i} x(k)+\sum_{i=1}^{r} h_{i}(k) \boldsymbol{E}_{i} \mu(k),
\end{aligned}
$$

where $h_{i}(k)=\omega_{i}(k) / \sum_{i=1}^{r} \omega_{i}(k), \omega_{i}(k)=\prod_{j=1}^{n_{x}} M_{i j}\left(x_{j}(k)\right)$ and $M_{i j}\left(x_{j}(k)\right)$ is the grade of membership of $x_{j}(k)$ in $M_{i j}$; $\omega_{i}(k)$ is the weight of the $\mathrm{i}$-th rule.

In some nonlinear systems, the system states usually cannot be completely measured. Therefore, the designers need to design the fuzzy observers to estimate the states for the fuzzy system in order to implement the fuzzy controller. In [18], the authors consider the socalled separation property for a controller and an observer for the linear stochastic systems. The fuzzy observers require to satisfy the condition $x(k)-\hat{x}(k) \rightarrow 0$ when $\mathrm{k} \rightarrow \infty$, where $\hat{x}(k)$ denotes the estimated state vector of the fuzzy observer. In this paper, the fuzzy observer is described as follows:

\section{Observer Rule i :}

IF $x_{1}(k)$ is $M_{i 1} \ldots$ and $x_{n_{x}}(k)$ is $M_{i n_{x}}$,

THEN $\hat{x}(k+1)=\boldsymbol{A}_{i} \hat{x}(k)+\boldsymbol{B}_{i} u(k)+\boldsymbol{K}_{i}(y(k)-\hat{y}(k))$,

$$
\hat{y}(k)=C_{i} \hat{x}(k), \quad i=1,2, \ldots, r,
$$

where $\boldsymbol{K}_{i} \in \Re^{n_{x} \times n_{y}}$ are observer gain matrices and $\hat{x}(k)$ $\in \Re^{n_{x}}$ is the state vector of observer. The $y(k)$ and $\hat{y}(k)$ are the output of the fuzzy system and the fuzzy observer, respectively. Then, the final estimated state and output of the fuzzy observer are characterized as follows.

$$
\begin{aligned}
& \hat{x}(k+1)= \sum_{i=1}^{r} h_{i}(k) \boldsymbol{A}_{i} \hat{x}(k)+\sum_{i=1}^{r} h_{i}(k) \boldsymbol{B}_{i} u(k) \\
&+\sum_{i=1}^{r} \sum_{j=1}^{r} h_{i}(k) h_{j}(k) \boldsymbol{K}_{i} \boldsymbol{C}_{j}[x(k)-\hat{x}(k)] \\
&+\sum_{i=1}^{r} \sum_{j=1}^{r} h_{i}(k) h_{j}(k) \boldsymbol{K}_{i} \boldsymbol{E}_{j} \mu(k), \\
& \hat{y}(k)=\sum_{i=1}^{r} h_{i}(k) \boldsymbol{C}_{i} \hat{x}(k),
\end{aligned}
$$

The same weight $h_{i}(k)$ of $\mathrm{i}$-th rule of the fuzzy system (2) and (3) is used for the fuzzy observer (5) and (6). 
The design parameters of the fuzzy observer are gain matrices $\boldsymbol{K}_{i}$ in each rule.

In this paper, the concept of PDC [25-27] is used to synthesize fuzzy control laws of observed-state feedback stabilization for the nonlinear systems, which are represented by discrete T-S type fuzzy stochastic models (1). The basic idea of the PDC approach is to design the feedback gains for each rule in the fuzzy models. Linear control design techniques can be used to design these linear controllers for each rule. Hence, the nonlinear system controller can be blended by local linear fuzzy controllers sharing the same fuzzy sets with the discrete T-S type fuzzy stochastic models (1). By using the observed state from the fuzzy observer, the feedback fuzzy controller becomes

\section{Observer-based Fuzzy Controller Rule i :}

IF $x_{1}(k)$ is $M_{i 1} \ldots$ and $x_{n_{x}}(k)$ is $M_{i n_{x}}$

THEN $u(k)=\boldsymbol{G}_{i} \hat{x}(k), i=1,2, \ldots, r$,

where $i=1,2, \ldots, r$ and $\mathrm{r}$ is the number of IF-THEN rule. The overall observed-state feedback fuzzy controller becomes

$$
u(k)=\sum_{i=1}^{r} h_{i}(k) \boldsymbol{G}_{i} \hat{x}(k) .
$$

This observed-state feedback fuzzy controller is nonlinear in general. By substituting (8) into (2) and (5), state and observer equations of the fuzzy system can be described as follows.

$$
\begin{aligned}
x(k+1) & =\sum_{i=1}^{r} h_{i}(k) \boldsymbol{A}_{i} x(k)+\sum_{i=1}^{r} \sum_{j=1}^{r} h_{i}(k) h_{j}(k) \boldsymbol{B}_{i} \boldsymbol{G}_{j} \hat{x}(k) \\
& +\sum_{i=1}^{r} h_{i}(k) \boldsymbol{D}_{i} v(k) \\
\hat{x}(k+1) & =\sum_{i=1}^{r} \sum_{j=1}^{r} h_{i}(k) h_{j}(k)\left(\boldsymbol{A}_{i}+\boldsymbol{B}_{i} \boldsymbol{G}_{j}\right) \hat{x}(k) \\
& +\sum_{i=1}^{r} \sum_{j=1}^{r} h_{i}(k) h_{j}(k) \boldsymbol{K}_{i} \boldsymbol{G}_{j}[x(k)-\hat{x}(k)] \\
& +\sum_{i=1}^{r} \sum_{j=1}^{r} h_{i}(k) h_{j}(k) \boldsymbol{K}_{i} \boldsymbol{E}_{j} \mu(k) .
\end{aligned}
$$

Introducing $\tilde{x}(k)=x(k)-\hat{x}(k), \boldsymbol{R}_{i j}=\frac{\left(\boldsymbol{A}_{i}+\boldsymbol{B}_{i} \boldsymbol{G}_{j}\right)+\left(\boldsymbol{A}_{j}+\boldsymbol{B}_{j} \boldsymbol{G}_{i}\right)}{2}$ and $\tilde{\boldsymbol{R}}_{i j}=\frac{\boldsymbol{B}_{i} \boldsymbol{G}_{j}+\boldsymbol{B}_{j} \boldsymbol{G}_{i}}{2}, i<j \leq r$, Eq. (9) can be rewritten as

$$
\begin{aligned}
x(k+1)= & \sum_{i=1}^{r} h_{i}(k) h_{i}(k)\left(\boldsymbol{A}_{i}+\boldsymbol{B}_{i} \boldsymbol{G}_{i}\right) x(k) \\
& +2 \sum_{i<j} h_{i}(k) h_{j}(k) \boldsymbol{R}_{i j} x(k) \\
& -\left[\sum_{i=1}^{r} h_{i}(k) h_{i}(k) \boldsymbol{B}_{i} \boldsymbol{G}_{i} \tilde{x}(k)+2 \sum_{i<1}^{r} h_{i}(k) h_{j}(k) \tilde{\boldsymbol{R}}_{i j} \tilde{x}(k)\right] \\
& +\sum_{i=1}^{r} h_{i}(k) \boldsymbol{D}_{i} v(k) .
\end{aligned}
$$

The observer error dynamics becomes

$$
\begin{aligned}
\tilde{x}(k+1) & =\sum_{i=1}^{r} h_{i}(k) h_{i}(k)\left(\boldsymbol{A}_{i}-\boldsymbol{K}_{i} \boldsymbol{C}_{i}\right) \tilde{x}(k) \\
& +2 \sum_{i<j} h_{i}(k) h_{j}(k) \boldsymbol{H}_{i j} \tilde{x}(k) \\
& -\left[\sum_{i=1}^{r} h_{i}(k) h_{i}(k) \boldsymbol{K}_{i} \boldsymbol{E}_{i} \boldsymbol{\mu}(k)+2 \sum_{i<1}^{r} h_{i}(k) h_{j}(k) \tilde{\boldsymbol{H}}_{i j} \mu(k)\right] \\
& +\sum_{i=1}^{r} h_{i}(k) \boldsymbol{D}_{i} v(k),
\end{aligned}
$$

where $\boldsymbol{H}_{i j}=\frac{\left(\boldsymbol{A}_{i}-\boldsymbol{K}_{i} \boldsymbol{C}_{j}\right)+\left(\boldsymbol{A}_{j}-\boldsymbol{K}_{j} \boldsymbol{C}_{i}\right)}{2}$ and $\tilde{\boldsymbol{H}}_{i j}=\frac{\boldsymbol{K}_{i} \boldsymbol{E}_{j}+\boldsymbol{K}_{j} \boldsymbol{E}_{i}}{2}$. Augmenting (11) and (12) yields:

$$
\begin{aligned}
\chi(k+1) & =\sum_{i=1}^{r} \sum_{k=1}^{r} h_{i}(k) h_{i}(k) h_{k}(k)\left[\boldsymbol{L}_{i} \chi(k)+\boldsymbol{N}_{i k} \bar{v}(k)\right] \\
& +2 \sum_{i<j}^{r} h_{i}(k) h_{j}(k)\left[\boldsymbol{L}_{i j} \chi(k)+\boldsymbol{N}_{i j} \bar{v}(k)\right],
\end{aligned}
$$

where

$$
\begin{aligned}
\chi(k) & =\left[\begin{array}{c}
x(k) \\
\tilde{x}(k)
\end{array}\right], \bar{v}(k)=\left[\begin{array}{l}
v(k) \\
\mu(k)
\end{array}\right], \\
\boldsymbol{L}_{i} & =\left[\begin{array}{cc}
\boldsymbol{A}_{i}+\boldsymbol{B}_{i} \boldsymbol{G}_{i}-\boldsymbol{B}_{i} \boldsymbol{G}_{i} \\
0 & \boldsymbol{A}_{i}-\boldsymbol{K}_{i} \boldsymbol{C}_{i}
\end{array}\right], \boldsymbol{L}_{i j}=\left[\begin{array}{cc}
\boldsymbol{R}_{i j}-\tilde{\boldsymbol{R}}_{i j} \\
0 & \boldsymbol{H}_{i j}
\end{array}\right], \\
\boldsymbol{N}_{i k} & =\left[\begin{array}{cc}
\boldsymbol{D}_{k} & 0 \\
\boldsymbol{D}_{k} & -\boldsymbol{K}_{i} \boldsymbol{E}_{i}
\end{array}\right], \boldsymbol{N}_{i j}=\left[\begin{array}{cc}
0 & 0 \\
0 & -\widetilde{\boldsymbol{H}}_{i j}
\end{array}\right] .
\end{aligned}
$$

If $\boldsymbol{L}_{i}$ is a stable matrix, the state covariance matrix $\boldsymbol{X}_{i}$ of each subsystem of (13) can be defined by [18, 21]

$$
\boldsymbol{X}_{i}=\lim _{k \rightarrow \infty} E\left[\chi(k) \chi(k)^{T}\right]
$$

Let the common covariance matrix for (13) be $\boldsymbol{X}$ such 
that

$$
\boldsymbol{X}=\boldsymbol{X}_{i}=\left[\begin{array}{c}
\boldsymbol{X}_{a a} \boldsymbol{X}_{a b} \\
\boldsymbol{X}_{a b}^{T} \boldsymbol{X}_{b b}
\end{array}\right], i=1,2, \ldots, r,
$$

and $\boldsymbol{X}=\boldsymbol{X}^{T}>0$, then $\boldsymbol{X}$ satisfies the following Lyapunov equation for each rule $[18,21]$ :

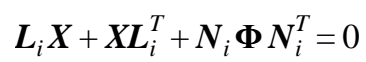

where $\boldsymbol{\Phi}=\left[\begin{array}{ll}\boldsymbol{V} & 0 \\ 0 & \boldsymbol{\Omega}\end{array}\right]$.

The purpose of this paper is to find the set of controllers $\boldsymbol{G}_{i}$ which satisfy the Lyapunov Eq. (16) such that the covariance matrix $\boldsymbol{X}_{a a}$ satisfies the following variance performance objectives:

$$
\lim _{k \rightarrow \infty} E\left[x_{\varphi}^{2}(k)\right]=\left[\boldsymbol{X}_{a a}\right]_{\varphi \varphi} \leq \sigma_{\varphi}^{2}, \varphi=1,2, \ldots, n_{x},
$$

where $\sigma_{\varphi}$ denote the Root-Mean-Squared (RMS) constraint for the variances of system states. This problem will be referred to as the variance constrained design using the CCOSF.

Based on the common covariance matrix defined in (15), references [20] provided the conditions and solutions for the optimal filter gains $\boldsymbol{K}_{i}$ as follows:

$$
\begin{aligned}
& \boldsymbol{K}_{i}=\boldsymbol{A}_{i} \boldsymbol{X}_{b b} \boldsymbol{C}_{i}^{T}\left(\boldsymbol{C}_{i} \boldsymbol{X}_{b b} \boldsymbol{C}_{i}^{T}+\boldsymbol{E}_{i} \boldsymbol{\Omega} \boldsymbol{E}_{i}^{T}\right)^{-1} \\
&\left(\boldsymbol{A}_{i}+\boldsymbol{B}_{i} \boldsymbol{G}_{i}\right) \boldsymbol{X}_{a a}\left(\boldsymbol{A}_{i}+\boldsymbol{B}_{i} \boldsymbol{G}_{i}\right)^{T} \\
& \quad-\boldsymbol{B}_{i} \boldsymbol{G}_{i} \boldsymbol{X}_{b b}\left(\boldsymbol{A}_{i}+\boldsymbol{B}_{i} \boldsymbol{G}_{i}\right)^{T}-\left(\boldsymbol{A}_{i}+\boldsymbol{B}_{i} \boldsymbol{G}_{i}\right) \boldsymbol{X}_{b b}\left(\boldsymbol{B}_{i} \boldsymbol{G}_{i}\right)^{T} \\
&+\boldsymbol{B}_{i} \boldsymbol{G}_{i} \boldsymbol{X}_{b b}\left(\boldsymbol{B}_{i} \boldsymbol{G}_{i}\right)^{T}+\boldsymbol{D}_{i} \boldsymbol{V} \boldsymbol{D}_{i}^{T}-\boldsymbol{X}_{a a}=0 \\
& \boldsymbol{X}_{b b}=\left(\boldsymbol{A}_{i}-\boldsymbol{K}_{i} \boldsymbol{C}_{i}\right) \boldsymbol{X}_{b b}\left(\boldsymbol{A}_{i}-\boldsymbol{K}_{i} \boldsymbol{C}_{i}\right)^{T} \\
& \quad+\boldsymbol{K}_{i}\left(\boldsymbol{E}_{i} \boldsymbol{\Omega} \boldsymbol{E}_{i}^{T}\right) \boldsymbol{K}_{i}^{T}+\boldsymbol{D}_{i} \boldsymbol{V} \boldsymbol{D}_{i}^{T}
\end{aligned}
$$

where $\boldsymbol{X}_{a a}>0, \boldsymbol{X}_{b b}>0$ and $\boldsymbol{X}_{a b}=\boldsymbol{X}_{b b}$ are defined in (15). Note that the assumption $\boldsymbol{X}_{a b}=\boldsymbol{X}_{b b}$ implies that the estimate $\hat{x}$ and the error $\tilde{x}$ are orthogonal, i.e., $E\left[\hat{x} \tilde{x}^{T}\right]=$ 0 . From the results of [20], it can be found that the optimal filter gain $\boldsymbol{K}_{i}=\boldsymbol{A}_{i} \boldsymbol{X}_{b b} \boldsymbol{C}_{i}^{T}\left(\boldsymbol{C}_{i} \boldsymbol{X}_{b b} \boldsymbol{C}_{i}^{T}+\boldsymbol{E}_{i} \boldsymbol{\Omega} \boldsymbol{E}_{i}^{T}\right)^{-1}$ leads to the fact that the steady state error between the system state $x(k)$ and the estimated state $\hat{x}(k)$ converges to zero when $k \rightarrow \infty$. Without loss of generality, this assumption has been applied in the design of optimal filter for the continuous-time systems [21,22] and discrete-time system [20], respectively.
From (18), note that if discrete T-S fuzzy model (1) is corrupted only by state noise without measurement noise (i.e., $\Omega=0$ ), then the optimal gain $\boldsymbol{K}_{i}$ does not exist. A variance constrained design methodology for discrete T-S fuzzy models, based on the theory of covariance control, has been developed in [8]. To offer a lucid presentation of the covariance control theory for discrete T-S fuzzy model (1), this paper recall the results of the stability of the whole system with the fuzzy observers. The CCOSF problem will be solved using the above optimal estimations (18-20) by the following theorem.

\section{Theorem 1}

Consider the fuzzy system (1) driven by (5) and (8) with the observer gain $\boldsymbol{K}_{i}$ defined in (18). If there exist common positive definite matrices $\boldsymbol{X}_{a a}>0, \boldsymbol{X}_{b b}>0$, $\boldsymbol{X}_{a b}=\boldsymbol{X}_{b b}$ and $\left(\boldsymbol{X}_{a a}=\boldsymbol{X}_{b b}\right)>0$ (as defined in (15)) satisfying the following conditions, then the equilibrium of the observed-state feedback fuzzy control system (11) is asymptotically stable in the large.

$$
\begin{aligned}
& \boldsymbol{A}_{i} \boldsymbol{X}_{b b} \boldsymbol{A}_{i}^{T}-\boldsymbol{A}_{i} \boldsymbol{X}_{b b} \boldsymbol{C}_{i}^{T}\left(\boldsymbol{C}_{i} \boldsymbol{X}_{b b} \boldsymbol{C}_{i}^{T}+\boldsymbol{E}_{i} \boldsymbol{\Omega} \boldsymbol{E}_{i}^{T}\right)^{-1} \boldsymbol{C}_{i} \boldsymbol{X}_{b b} \boldsymbol{A}_{i}^{T} \\
& \quad+\boldsymbol{D}_{i} \boldsymbol{V} \boldsymbol{D}_{i}^{T}-\boldsymbol{X}_{b b}=0 \\
& \left(\boldsymbol{A}_{i}+\boldsymbol{B}_{i} \boldsymbol{G}_{i}\right)\left(\boldsymbol{X}_{a a}-\boldsymbol{X}_{b b}\right)\left(\boldsymbol{A}_{i}+\boldsymbol{B}_{i} \boldsymbol{G}_{i}\right)^{T} \\
& \quad+\boldsymbol{A}_{i} \boldsymbol{X}_{b b} \boldsymbol{C}_{i}^{T}\left(\boldsymbol{C}_{i} \boldsymbol{X}_{b b} \boldsymbol{C}_{i}^{T}+\boldsymbol{E}_{i} \boldsymbol{\Omega} \boldsymbol{E}_{i}^{T}\right)^{-1} \boldsymbol{C}_{i} \boldsymbol{X}_{b b} \boldsymbol{A}_{i}^{T} \\
& \quad-\left(\boldsymbol{X}_{a a}-\boldsymbol{X}_{b b}\right)=0 \\
& \boldsymbol{R}_{i j}\left(\boldsymbol{X}_{a a}-\boldsymbol{X}_{b b}\right) \boldsymbol{R}_{i j}^{T}-\left(\boldsymbol{X}_{a a}-\boldsymbol{X}_{b b}\right)<0, i<j \leq r
\end{aligned}
$$

\section{Proof:}

From the previous statements, it is clear that the matrix $\boldsymbol{K}_{i}$ performs an optimal filter gain if and only if there exist matrices $\boldsymbol{X}_{a a}>0, \boldsymbol{X}_{b b}>0$ such that (18-20) are all satisfied with $\boldsymbol{X}_{a b}=\boldsymbol{X}_{b b}$ defined in (15). Substituting (18) into (20) and rearranging yields

$$
\begin{aligned}
\boldsymbol{X}_{b b}= & \boldsymbol{A}_{i} \boldsymbol{X}_{b b} \boldsymbol{A}_{i}^{T}-\boldsymbol{A}_{i} \boldsymbol{X}_{b b} \boldsymbol{C}_{i}^{T}\left(\boldsymbol{C}_{i} \boldsymbol{X}_{b b} \boldsymbol{C}_{i}^{T}+\boldsymbol{E}_{i} \boldsymbol{\Omega} \boldsymbol{E}_{i}^{T}\right)^{-1} \\
& \boldsymbol{C}_{i} \boldsymbol{X}_{b b} \boldsymbol{A}_{i}^{T}+\boldsymbol{D}_{i} \boldsymbol{V D _ { i } { } ^ { T }}
\end{aligned}
$$

which is equivalent to (21). Putting (24) into (19), then one can obtain

$$
\begin{aligned}
\boldsymbol{X}_{a a}-\boldsymbol{X}_{b b} & =\left(\boldsymbol{A}_{i}+\boldsymbol{B}_{i} \boldsymbol{G}_{i}\right)\left(\boldsymbol{X}_{a a}-\boldsymbol{X}_{b b}\right)\left(\boldsymbol{A}_{i}+\boldsymbol{B}_{i} \boldsymbol{G}_{i}\right)^{T} \\
& +\boldsymbol{A}_{i} \boldsymbol{X}_{b b} \boldsymbol{C}_{i}^{T}\left(\boldsymbol{C}_{i} \boldsymbol{X}_{b b} \boldsymbol{C}_{i}^{T}+\boldsymbol{E}_{i} \boldsymbol{\Omega} \boldsymbol{E}_{i}^{T}\right)^{-1} \boldsymbol{C}_{i} \boldsymbol{X}_{b b} \boldsymbol{A}_{i}^{T}
\end{aligned}
$$


Thus, the conditions (19) and (20) can be replaced by (23) and (21) with the observer gain $\boldsymbol{K}_{i}$ defined in (18). From [20], it can be found that the optimal filter gain $\boldsymbol{K}_{i}$ defined in (18) satisfying (19-20) or (21-22) leads to the fact that the steady state error of $\tilde{x}(k)$ approximates to zero when $k \rightarrow \infty$. From Theorem 3 of [25], one can find that if there exist a common positive definite error state covariance matrix $\left(\boldsymbol{X}_{a a}-\boldsymbol{X}_{b b}\right)$ satisfying (22) and (23), then the equilibrium of continuous fuzzy control system (11) is asymptotically stable in the large due to $\boldsymbol{A}_{i} \boldsymbol{X}_{b b} \boldsymbol{C}_{i}^{T}$ $\left(\boldsymbol{C}_{i} \boldsymbol{X}_{b b} \boldsymbol{C}_{i}^{T}+\boldsymbol{E}_{i} \boldsymbol{\Omega} \boldsymbol{E}_{i}^{T}\right)^{-1} \boldsymbol{C}_{i} \boldsymbol{X}_{b b} \boldsymbol{A}_{i}^{T} \geq 0$ and $\widetilde{x}(k) \rightarrow 0$. Hence, it can be concluded that if conditions (21-23) are satisfied with the observer gain $\boldsymbol{K}_{i}$ defined in (18), then the equilibrium of the observed-state feedback fuzzy control stochastic system (11) is asymptotically stable in the large.

From Theorem 1, the main purpose of this paper is to solve the control feedback gain matrices $\boldsymbol{G}_{i}$ such that the designers can directly assign common positive definite error state covariance matrix $\left(\boldsymbol{X}_{a a}-\boldsymbol{X}_{b b}\right)$ to achieve the variance constraints (17). Achieving the above stability conditions requires the control feedback gains $\boldsymbol{G}_{i}$ that satisfy (21-23) so that the closed-loop fuzzy system (11) will be asymptotically stable. The next section shows how to assign the common covariance matrix $\boldsymbol{X}$ and then find control feedback gains $\boldsymbol{G}_{i}$ by using the theory of generalized inverse.

\section{THE SOLUTIONS OF OBSERVED-STATE FEEDBACK GAINS FOR DISCRETE T-S TYPE FUZZY CONTROLLERS}

In this section, the results of above section will be applied to develop a method for solving $\boldsymbol{G}_{i}$ subject to the assigned common covariance matrix $\boldsymbol{X}$. Stability conditions of Theorem 1 and the solutions of CCOSF problems are discussed in the following theorem.

\section{Theorem 2}

Consider the discrete T-S fuzzy model (1) driven by (5) and (8) with the observer gain $\boldsymbol{K}_{i}=\boldsymbol{A}_{i} \boldsymbol{X}_{b b} \boldsymbol{C}_{i}^{T}$ $\left(\boldsymbol{C}_{i} \boldsymbol{X}_{b b} \boldsymbol{C}_{i}^{T}+\boldsymbol{E}_{i} \boldsymbol{\Omega} \boldsymbol{E}_{i}^{T}\right)^{-1}$, where $\boldsymbol{X}_{b b}>0$ satisfies Eq. (21). It is assumed that the factor of $\left(\boldsymbol{X}_{a a}-\boldsymbol{X}_{b b}\right)$, where $\boldsymbol{X}_{a a}$ and $\boldsymbol{X}_{b b}$ are defined in (15), is $\mathbf{F}$ (i.e., $\boldsymbol{F F}^{T}=\left(\boldsymbol{X}_{a a}-\boldsymbol{X}_{b b}\right)$ and the matrices $\boldsymbol{H}_{i}$ and $\boldsymbol{L}_{i}$ are defined by

$$
\begin{aligned}
\boldsymbol{H}_{i} & =\left(\boldsymbol{I}-\boldsymbol{B}_{i} \boldsymbol{B}_{i}^{+}\right)\left(\boldsymbol{X}_{a a}-\boldsymbol{X}_{b b}-\boldsymbol{A}_{i} \boldsymbol{X}_{b b} \boldsymbol{C}_{i}^{T}\left(\boldsymbol{C}_{i} \boldsymbol{X}_{b b} \boldsymbol{C}_{i}^{T}\right.\right. \\
& \left.\left.+\boldsymbol{E}_{i} \boldsymbol{\Omega} \boldsymbol{E}_{i}^{T}\right)^{-1} \boldsymbol{C}_{i} \boldsymbol{X}_{b b} \boldsymbol{A}_{i}^{T}\right)^{\frac{1}{2}} \\
\boldsymbol{L}_{i} & =\left(\boldsymbol{I}-\boldsymbol{B}_{i} \boldsymbol{B}_{i}^{+}\right) \boldsymbol{A}_{i} \boldsymbol{F}
\end{aligned}
$$

where $[\cdot]^{+}$denotes the Moore-Penrose inverse of $[\cdot]$, $[\cdot]^{\frac{1}{2}}$ is the unique positive semi-definite square root of [·] and the matrices $\boldsymbol{H}_{i}$ and $\boldsymbol{L}_{i}$ have rank $m_{r}$ and the following singular value decompositions.

$$
\begin{gathered}
\boldsymbol{H}_{i}=\boldsymbol{\Gamma}_{i} \Sigma_{i} \boldsymbol{Q}_{i}^{T} \\
\boldsymbol{L}_{i}=\Gamma_{i} \Sigma_{i} \boldsymbol{T}_{i}^{T}
\end{gathered}
$$

where $\Gamma_{i}, \boldsymbol{Q}_{i}, \boldsymbol{T}_{i}$ are orthonormal, $\Sigma=\operatorname{diag}\left(\sigma_{1}, \ldots, \sigma_{n_{x}}\right)$, $\sigma_{1} \geq \sigma_{2} \geq \ldots \geq \sigma_{m_{r}}>0=\sigma_{m_{r}+1}=\ldots=\sigma_{n_{x}}$.

Then the system has observed-state feedback gains $\boldsymbol{G}_{i}$ that achieve stability condition (22) for a common positive definite error state covariance $\left(\boldsymbol{X}_{a a}-\boldsymbol{X}_{b b}\right)>0$ if and only if the following condition is satisfied.

$$
\begin{aligned}
& \boldsymbol{X}_{a a} \geq \boldsymbol{X}_{b b}+\boldsymbol{A}_{i} \boldsymbol{X}_{b b} \boldsymbol{C}_{i}^{T}\left(\boldsymbol{C}_{i} \boldsymbol{X}_{b b} \boldsymbol{C}_{i}^{T}+\boldsymbol{E}_{i} \boldsymbol{\Omega} \boldsymbol{E}_{i}^{T}\right)^{-1} \boldsymbol{C}_{i} \boldsymbol{X}_{b b} \boldsymbol{A}_{i}^{T} \\
& \left(\boldsymbol{I}-\boldsymbol{B}_{i} \boldsymbol{B}_{i}^{+}\right)\left(\boldsymbol{A}_{i} \boldsymbol{X}_{a a} \boldsymbol{A}_{i}^{T}-\boldsymbol{X}_{a a}+\boldsymbol{D}_{i} \boldsymbol{V} \boldsymbol{D}_{i}^{T}\right)\left(\boldsymbol{I}-\boldsymbol{B}_{i} \boldsymbol{B}_{i}^{+}\right)=0
\end{aligned}
$$

Moreover, assume that the conditions (30) and (31) are all satisfied, and then the set of all convenient observed-state feedback gains $\boldsymbol{G}_{i}$ that solve CCOSF problem is given by

$$
\begin{gathered}
\boldsymbol{G}_{i}=\boldsymbol{B}_{i}^{+}\left\{\left(\boldsymbol{X}_{a a}-\boldsymbol{X}_{b b}-\boldsymbol{A}_{i} \boldsymbol{X}_{b b} \boldsymbol{C}_{i}^{T}\left(\boldsymbol{C}_{i} \boldsymbol{X}_{b b} \boldsymbol{C}_{i}^{T}+\boldsymbol{E}_{i} \boldsymbol{\Omega} \boldsymbol{E}_{i}^{T}\right)^{-1}\right.\right. \\
\left.\left.\boldsymbol{C}_{i} \boldsymbol{X}_{b b} \boldsymbol{A}_{i}^{T}\right)^{\frac{1}{2}} \boldsymbol{S}_{i} \boldsymbol{F}^{-1}-\boldsymbol{A}_{i}\right\}+\left(\boldsymbol{I}_{n_{u}}-\boldsymbol{B}_{i}^{+} \boldsymbol{B}_{i}\right) \boldsymbol{Y}_{i},
\end{gathered}
$$

where $\boldsymbol{Y}_{i} \in \Re^{n_{u} \times n_{x}}$ is arbitrary (note $\boldsymbol{Y}_{i}=0$ is such arbitrary) and $\boldsymbol{S}_{i} \in \widetilde{\boldsymbol{S}}_{i}$. The set $\widetilde{\boldsymbol{S}}_{i}$ is expressed as

$$
\begin{aligned}
& \tilde{S}_{i}=\left\{\boldsymbol{S}_{i}: \boldsymbol{S}_{i}=\boldsymbol{Q}_{i}\left[\begin{array}{cc}
\boldsymbol{I}_{r} & 0 \\
0 & \boldsymbol{U}_{0}
\end{array}\right] \boldsymbol{T}_{i}^{T}, \boldsymbol{U}_{0} \in \Re^{\left(n_{x}-m_{r}\right) \times\left(n_{x}-m_{r}\right)}\right. \\
& \text { is arbitrarily orthonormal }
\end{aligned}
$$

\section{Proof:}

\section{Necessity}

Suppose there exists an observed-state feedback 
gain $\boldsymbol{G}_{i}$ satisfies

$$
\begin{aligned}
\boldsymbol{A}_{i}+\boldsymbol{B}_{i} \boldsymbol{G}_{i} & =\left(\boldsymbol{X}_{a a}-\boldsymbol{X}_{b b}-\boldsymbol{A}_{i} \boldsymbol{X}_{b b} \boldsymbol{C}_{i}^{T}\left(\boldsymbol{C}_{i} \boldsymbol{X}_{b b} \boldsymbol{C}_{i}^{T}\right.\right. \\
& \left.\left.+\boldsymbol{E}_{i} \boldsymbol{\Omega} \boldsymbol{E}_{i}^{T}\right)^{-1} \boldsymbol{C}_{i} \boldsymbol{X}_{b b} \boldsymbol{A}_{i}^{T}\right)^{\frac{1}{2}} \boldsymbol{S}_{i} \boldsymbol{F}^{-1}
\end{aligned}
$$

or

$$
\begin{aligned}
\boldsymbol{B}_{i} \boldsymbol{G}_{i} & =\left(\boldsymbol{X}_{a a}-\boldsymbol{X}_{b b}-\boldsymbol{A}_{i} \boldsymbol{X}_{b b} \boldsymbol{C}_{i}^{T}\left(\boldsymbol{C}_{i} \boldsymbol{X}_{b b} \boldsymbol{C}_{i}^{T}\right.\right. \\
& \left.\left.+\boldsymbol{E}_{i} \boldsymbol{\Omega} \boldsymbol{E}_{i}^{T}\right)^{-1} \boldsymbol{C}_{i} \boldsymbol{X}_{b b} \boldsymbol{A}_{i}^{T}\right)^{\frac{1}{2}} \boldsymbol{S}_{i} \boldsymbol{F}^{-1}-\boldsymbol{A}_{i}
\end{aligned}
$$

where $S_{i}$ is some orthonormal matrix. From the wellknow results of the generalized inverse theory [1], (34) has a solution $\boldsymbol{G}_{i}$ if and only if

$$
\begin{gathered}
\boldsymbol{B}_{i} \boldsymbol{B}_{i}^{+}\left(\left(\boldsymbol{X}_{a a}-\boldsymbol{X}_{b b}-\boldsymbol{A}_{i} \boldsymbol{X}_{b b} \boldsymbol{C}_{i}^{T}\left(\boldsymbol{C}_{i} \boldsymbol{X}_{b b} \boldsymbol{C}_{i}^{T}\right.\right.\right. \\
\left.\left.\left.+\boldsymbol{E}_{i} \boldsymbol{\Omega} \boldsymbol{E}_{i}^{T}\right)^{-1} \boldsymbol{C}_{i} \boldsymbol{X}_{b b} \boldsymbol{A}_{i}^{T}\right)^{\frac{1}{2}} \boldsymbol{S}_{i} \boldsymbol{F}^{-1}-\boldsymbol{A}_{i}\right) \\
=\left(\boldsymbol{X}_{a a}-\boldsymbol{X}_{b b}-\boldsymbol{A}_{i} \boldsymbol{X}_{b b} \boldsymbol{C}_{i}^{T}\left(\boldsymbol{C}_{i} \boldsymbol{X}_{b b} \boldsymbol{C}_{i}^{T}\right.\right. \\
\left.\left.+\boldsymbol{E}_{i} \boldsymbol{\Omega} \boldsymbol{E}_{i}^{T}\right)^{-1} \boldsymbol{C}_{i} \boldsymbol{X}_{b b} \boldsymbol{A}_{i}^{T}\right)^{\frac{1}{2}} \boldsymbol{S}_{i} \boldsymbol{F}^{-1}-\boldsymbol{A}_{i}
\end{gathered}
$$

or

$$
\begin{aligned}
(\boldsymbol{I} & \left.-\boldsymbol{B}_{i} \boldsymbol{B}_{i}^{+}\right)\left(\boldsymbol{X}_{a a}-\boldsymbol{X}_{b b}-\boldsymbol{A}_{i} \boldsymbol{X}_{b b} \boldsymbol{C}_{i}^{T}\left(\boldsymbol{C}_{i} \boldsymbol{X}_{b b} \boldsymbol{C}_{i}^{T}\right.\right. \\
& \left.\left.+\boldsymbol{E}_{i} \boldsymbol{\Omega} \boldsymbol{E}_{i}^{T}\right)^{-1} \boldsymbol{C}_{i} \boldsymbol{X}_{b b} \boldsymbol{A}_{i}^{T}\right) \frac{1}{2} \boldsymbol{S}_{i}=\left(\boldsymbol{I}-\boldsymbol{B}_{i} \boldsymbol{B}_{i}^{+}\right) \boldsymbol{A}_{i} \boldsymbol{F}
\end{aligned}
$$

Then, it is well known that (34) is consistent, if and only if there exists an orthonormal matrix $\boldsymbol{S}_{i}$ which satisfies

$$
\boldsymbol{H}_{i} \boldsymbol{S}_{i}=\boldsymbol{L}_{i}
$$

where $\boldsymbol{H}_{i}$ and $\boldsymbol{L}_{i}$ are defined in (26) and (27).

Now, assume that $\boldsymbol{S}_{i}$ is orthonormal and multiply (36) by its transpose to obtain

$$
\boldsymbol{H}_{i} \boldsymbol{H}_{i}^{T}=\boldsymbol{L}_{i} \boldsymbol{L}_{i}^{T}
$$

Then, (37) is a necessary condition for the existence of $\boldsymbol{G}_{i}$.

\section{Sufficiency}

By assuming (37), this proof wants to show that (36) is true. Note that the symmetric, positive semidefinite matrix $\boldsymbol{L}_{i} \boldsymbol{L}_{i}^{T}$ of rank $m_{r}$ may be expressed as

$$
\boldsymbol{L}_{i} \boldsymbol{L}_{i}^{T}=\boldsymbol{\Gamma}_{i} \boldsymbol{\Sigma}_{i}^{2} \boldsymbol{\Gamma}_{i}^{T}
$$

where $\Gamma_{i}$ is orthonormal, $\Sigma=\operatorname{diag}\left(\sigma_{1}, \ldots, \sigma_{n_{x}}\right)$, and $\sigma_{1} \geq \sigma_{2}$ $\geq \ldots \geq \sigma_{m_{r}}>0=\sigma_{m_{r}+1}=\ldots=\sigma_{n_{x}}$. Therefore, if (37) is satisfied, then

$$
\boldsymbol{H}_{i} \boldsymbol{H}_{i}^{T}=\boldsymbol{\Gamma}_{i} \boldsymbol{\Sigma}_{i}^{2} \boldsymbol{\Gamma}_{i}^{T}
$$

Thus, $\boldsymbol{H}_{i}$ and $\boldsymbol{L}_{i}$ may be expressed as (28) and (29) by means of singular value decomposition.

Now, substitute (28) and (29) into (36) to obtain

$$
\boldsymbol{\Gamma}_{i} \boldsymbol{\Sigma}_{i} \boldsymbol{Q}_{i}^{T} \boldsymbol{S}_{i}=\boldsymbol{\Gamma}_{i} \boldsymbol{\Sigma}_{i} \boldsymbol{T}_{i}^{T}
$$

Then, it is seen that (40) admits an orthonormal solution

$$
S_{i}=Q_{i} T_{i}^{T}
$$

Thus, if (37) is assumed, the orthonormal matrix $\boldsymbol{S}_{i}$ defined by (41) satisfies (36). Consequently, the observed-state feedback gains $\boldsymbol{G}_{i}$, which satisfies (34), will exist, if and only if (37) is true. By substituting (26) and (27) into (37), it is easily seen that (37) is equivalent to (31).

Moreover, the solution of the present problem will be obtained as follows. Substituting (28) and (29) into (36) gives

$$
\Gamma_{i} \Sigma_{i} Q_{i}^{T} S_{i}=\Gamma_{i} \Sigma_{i} T_{i}^{T}
$$

Also, premultiplying (42) by $\boldsymbol{Q}_{i} \boldsymbol{\Gamma}_{i}^{T}$ gives

$$
\boldsymbol{Q}_{i} \boldsymbol{\Sigma}_{i} \boldsymbol{Q}_{i}^{T} \boldsymbol{S}_{i}=\boldsymbol{Q}_{i} \boldsymbol{\Sigma}_{i} \boldsymbol{T}_{i}^{T}
$$

Since $\boldsymbol{Q}_{i} \boldsymbol{\Gamma}_{i}^{T}$ is nonsingular, (42) and (43) are equivalent statements, so $S_{i}$ must be an orthonormal solution of (43). However, by comparing (43) with Lemma A1 and Lemma A2, it is seen that $S_{i}$ is an orthonormal matrix in the polar decomposition of the matrix $\boldsymbol{J}_{i}=\boldsymbol{Q}_{i} \Sigma_{i} \boldsymbol{T}_{i}^{T}$. Thus, using Lemma A2, $\boldsymbol{S}_{i}$ satisfies (43) (or equivalently, the condition (36)), if and only if $\boldsymbol{S}_{i} \in \tilde{\boldsymbol{S}}_{i}$.

For any $\boldsymbol{S}_{i} \in \widetilde{\boldsymbol{S}}_{i}$, the observed-state feedback gain $\boldsymbol{G}_{i}$ is a solution of (34), if and only if it may be expressed as [1]

$$
\begin{aligned}
\boldsymbol{G}_{i} & =\boldsymbol{B}_{i}^{+}\left\{\left(\boldsymbol{X}_{a a}-\boldsymbol{X}_{b b}-\boldsymbol{A}_{i} \boldsymbol{X}_{b b} \boldsymbol{C}_{i}^{T}\left(\boldsymbol{C}_{i} \boldsymbol{X}_{b b} \boldsymbol{C}_{i}^{T}\right.\right.\right. \\
& \left.\left.+\boldsymbol{E}_{i} \boldsymbol{\Omega} \boldsymbol{E}_{i}^{T}\right)^{-1} \boldsymbol{C}_{i} \boldsymbol{X}_{b b} \boldsymbol{A}_{i}^{T} \frac{1}{2} \boldsymbol{S}_{i} \boldsymbol{F}^{-1}-\boldsymbol{A}_{i}\right\} \\
& +\left(\boldsymbol{I}_{n_{u}}-\boldsymbol{B}_{i}^{+} \boldsymbol{B}_{i}\right) \boldsymbol{Y}_{i}
\end{aligned}
$$


for some $\boldsymbol{Y}_{i} \in \Re^{n_{u} \times n_{x}}$.

Theorem 2 provides the conditions and solutions for the existence of observed-state feedback gains $\boldsymbol{G}_{i}$ such that the stability condition (22) is satisfied. From the above results, the design steps for the constrained variance design procedure of the CCOSF fuzzy controller design problems can be summarized as follows.

Step 1. Solve the positive definite matrix $\boldsymbol{X}_{b b}$ from algebraic Riccati-like Eq. (21).

Step 2. Assign the diagonal elements of matrix $\boldsymbol{X}_{a a}$ to satisfy $\left[\boldsymbol{X}_{b b}\right]_{\varphi \varphi} \leq\left[\boldsymbol{X}_{a a}\right]_{\varphi \varphi} \leq \sigma_{\varphi}^{2}, \varphi=1,2, \ldots, n_{X}$, which can guarantee that the constraint (17) is satisfied.

Step 3. Use variance constrained design methodology [12] to solve the off-diagonal elements of $\boldsymbol{X}_{a a}$ from (31).

Step 4. If the matrix $\boldsymbol{X}_{a a}$ solved from Step 3 does not satisfy condition $\boldsymbol{X}_{a a}-\boldsymbol{X}_{b b}-\boldsymbol{A}_{i} \boldsymbol{X}_{b b} \boldsymbol{C}_{i}^{T}\left(\boldsymbol{C}_{i} \boldsymbol{X}_{b b} \boldsymbol{C}_{i}^{T}+\right.$ $\left.\boldsymbol{E}_{i} \boldsymbol{\Omega} \boldsymbol{E}_{i}^{T}\right)^{-1} \boldsymbol{C}_{i} \boldsymbol{X}_{b b} \boldsymbol{A}_{i}^{T} \geq 0$, then go to Step 2; otherwise, continues.

Step 5. Substitute $\boldsymbol{X}_{a a}$ and $\boldsymbol{X}_{b b}$ into (18) and (32) to obtain optimal filter gains $\boldsymbol{K}_{i}$ and observedstated feedback gains $\boldsymbol{G}_{i}$, respectively.

Step 6. Substitute $\boldsymbol{X}_{a a}, \boldsymbol{X}_{b b}$ and $\boldsymbol{G}_{i}$ into (23) to check whether (23) is satisfied. If (23) is not satisfied, it is necessary to go to Step 2 to reassign common state covariance matrix $\boldsymbol{X}_{a a}$.

In the following section, the application of the variance constrained design approach with observerbased fuzzy covariance controllers will be illustrated by a numerical example.

\section{A NUMERICAL EXAMPLE}

To design the fuzzy controller and the fuzzy observer, it is necessary to construct a T-S type fuzzy model, which represents the dynamics of a nonlinear stochastic plant. In this section, a nonlinear discrete stochastic system is considered as follows:

$$
\begin{aligned}
x_{1}(k+1) & =x_{2}(k), \\
x_{2}(k+1)= & \left(-\sqrt{2}+\frac{\pi}{2.39} \cos x_{1}(k)\right) \sin x_{1}(k) \\
& +\left(1.25+3.75 \cos x_{1}(k)\right) x_{2}(k) \\
& -\frac{1}{3.144-0.177 \cos x_{1}(k)} u(k) \\
& +\left(0.03+0.0355 \cos x_{1}(k)\right) v(k), \\
y(k)=- & 0.35 x_{1}(k)-0.0025 \mu(k),
\end{aligned}
$$

where the covariance matrices of zero-mean white noises $v(k)$ and $\mu(k)$ are $\mathrm{V}=0.2$ and $\Omega=0.1$, respectively. It is assumed that the range of nonlinear state variable $x_{1}(k)$ is $x_{1}(k) \in\left(-\frac{\pi}{2}, \frac{\pi}{2}\right)$. The nonlinear system (45) can be represented by the following two-rule (i.e., $r=2$ ) T-S fuzzy model [26].

\section{Plant Rule ${ }^{1}$ :}

IF $x_{1}(k)$ is about 0

$$
\begin{gathered}
\text { THEN } \dot{x}(k)=\boldsymbol{A}_{1} x(k)+\boldsymbol{B}_{1} u(k)+\boldsymbol{D}_{1} v(k) \\
y(k)=\boldsymbol{C}_{1} x(k)+\boldsymbol{E}_{1} \boldsymbol{\mu}(k)
\end{gathered}
$$

\section{Plant Rule ${ }^{2}$ :}

IF $x_{1}(k)$ is about $\pm \frac{\pi}{2}\left(\left|x_{1}\right|<\frac{\pi}{2}\right)$

$$
\begin{gathered}
\text { THEN } \dot{x}(k)=\boldsymbol{A}_{2} x(k)+\boldsymbol{B}_{2} u(k)+\boldsymbol{D}_{2} v(k) \\
y(k)=\boldsymbol{C}_{2} x(k)+\boldsymbol{E}_{2} \boldsymbol{\mu}(k)
\end{gathered}
$$

where

$$
\begin{aligned}
& \boldsymbol{A}_{1}=\left[\begin{array}{cc}
0 & 1 \\
-0.1 & 5
\end{array}\right], \boldsymbol{A}_{2}=\left[\begin{array}{cc}
0 & 1 \\
-0.9 & 1.25
\end{array}\right], \\
& \boldsymbol{B}_{1}=\left[\begin{array}{c}
0 \\
-0.337
\end{array}\right], \boldsymbol{B}_{2}=\left[\begin{array}{c}
0 \\
-0.318
\end{array}\right], \boldsymbol{C}_{1}=\boldsymbol{C}_{2}=\left[\begin{array}{ll}
0 & -0.35
\end{array}\right], \\
& \boldsymbol{D}_{1}=\left[\begin{array}{c}
0 \\
0.0655
\end{array}\right], \boldsymbol{D}_{2}=\left[\begin{array}{c}
0 \\
0.03
\end{array}\right], \boldsymbol{E}_{1}=\boldsymbol{E}_{2}=-0.0025,
\end{aligned}
$$

Figure 1 shows the membership functions of nonlinear state variable $x_{1}(k)$ of T-S fuzzy model (46). In Figure 1, the fuzzy sets for fuzzy rules are described by two triangular membership functions. In this numerical example, it is assumed that the constraints for state variances of the nonlinear system (45) are

$$
\left[\boldsymbol{X}_{a a}\right]_{11} \leq 0.2,\left[\boldsymbol{X}_{a a}\right]_{22} \leq 0.175
$$

According to Step 1 of the design procedure, the positive definite matrix $\boldsymbol{X}_{b b}$ can be obtained by solving the algebraic Riccati-like Eq. (21).

$$
\boldsymbol{X}_{b b}=\left[\begin{array}{cc}
0.001 & 0 \\
0 & 0.003
\end{array}\right]
$$




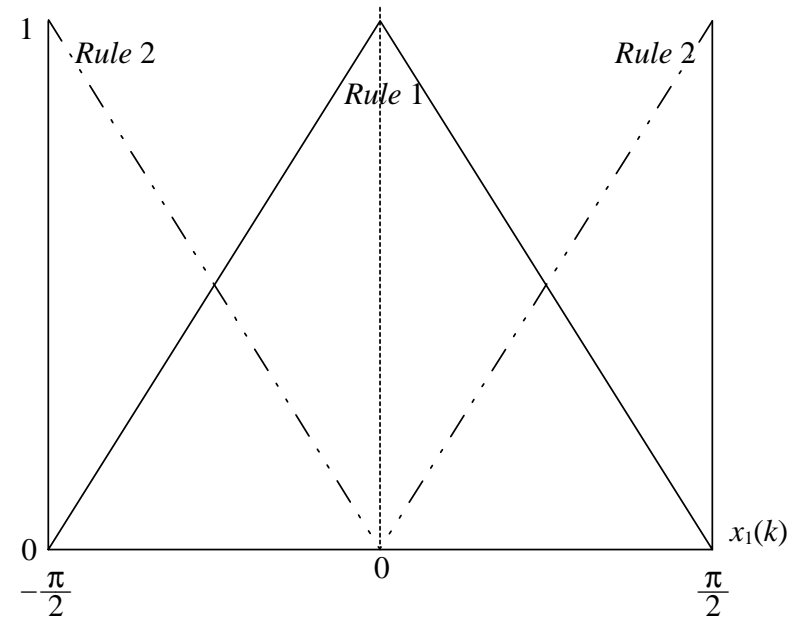

Fig. 1. The membership function of $x_{1}(k)$.

From Step 2, we first assign the diagonal entries of $\boldsymbol{X}_{a a}$ as $\left[\boldsymbol{X}_{a a}\right]_{11}=0.15$ and $\left[\boldsymbol{X}_{a a}\right]_{22}=0.15$ such that the variance constraints (47) are satisfied. Next, applying the variance constrained design methodology [12] to solve the off-diagonal elements of $\boldsymbol{X}_{a a}$ from (31) yields

$$
\boldsymbol{X}_{a a}=\left[\begin{array}{ll}
0.15 & 0.05 \\
0.05 & 0.15
\end{array}\right] .
$$

Putting the matrices $\boldsymbol{X}_{a a}$ and $\boldsymbol{X}_{b b}$ into (15), the common covariance matrix $\boldsymbol{X}$ becomes

$$
\boldsymbol{X}=\left[\begin{array}{cccc}
0.15 & 0.05 & 0.001 & 0 \\
0.05 & 0.15 & 0 & 0.003 \\
0.001 & 0 & 0.001 & 0 \\
0 & 0.003 & 0 & 0.003
\end{array}\right]
$$

Subtracting $\boldsymbol{X}_{b b}$ from $\boldsymbol{X}_{a a}$, one can obtain

$$
\begin{aligned}
& \boldsymbol{X}_{a a}-\boldsymbol{X}_{b b}-\boldsymbol{A}_{1} \boldsymbol{X}_{b b} \boldsymbol{C}_{1}^{T}\left(\boldsymbol{C}_{1} \boldsymbol{X}_{b b} \boldsymbol{C}_{1}^{T}\right. \\
& \left.+\boldsymbol{E}_{1} \boldsymbol{\Omega} \boldsymbol{E}_{1}^{T}\right)^{-1} \boldsymbol{C}_{1} \boldsymbol{X}_{b b} \boldsymbol{A}_{1}^{T}=\left[\begin{array}{ll}
0.1460 & 0.0350 \\
0.0350 & 0.0721
\end{array}\right] \geq 0 . \\
& \boldsymbol{X}_{a a}-\boldsymbol{X}_{b b}-\boldsymbol{A}_{2} \boldsymbol{X}_{b b} \boldsymbol{C}_{2}^{T}\left(\boldsymbol{C}_{2} \boldsymbol{X}_{b b} \boldsymbol{C}_{2}^{T}\right. \\
& \left.+\boldsymbol{E}_{2} \boldsymbol{\Omega} \boldsymbol{E}_{2}^{T}\right)^{-1} \boldsymbol{C}_{2} \boldsymbol{X}_{b b} \boldsymbol{A}_{2}^{T}=\left[\begin{array}{ll}
0.1460 & 0.0463 \\
0.0463 & 0.1423
\end{array}\right] \geq 0 .
\end{aligned}
$$

According to Step 5, the optimal filter gains $\boldsymbol{K}_{i}$ and observed-stated feedback gains $\boldsymbol{G}_{i}$ can be obtained by substituting $\boldsymbol{X}_{a a}$ and $\boldsymbol{X}_{b b}$ into (18) and (32), respectively.

$$
\boldsymbol{K}_{1}=\left[\begin{array}{ll}
-2.852 & -14.261
\end{array}\right]^{T}, \boldsymbol{K}_{2}=\left[\begin{array}{ll}
-2.852 & -3.565
\end{array}\right]^{T}
$$

$$
\boldsymbol{G}_{1}=\left[\begin{array}{ll}
1.765 & 13.426
\end{array}\right], \boldsymbol{G}_{2}=\left[\begin{array}{ll}
0.262 & 1.886
\end{array}\right]
$$

Finally, substituting $\boldsymbol{X}_{a a}, \boldsymbol{X}_{b b}$ and $\boldsymbol{G}_{i}$ into (23) yields

$$
\begin{gathered}
\boldsymbol{R}_{i j}\left(\boldsymbol{X}_{a a}-\boldsymbol{X}_{b b}\right) \boldsymbol{R}_{i j}^{T}-\left(\boldsymbol{X}_{a a}-\boldsymbol{X}_{b b}\right) \\
\quad=\left[\begin{array}{l}
-0.0020-0.0076 \\
-0.0076-0.0346
\end{array}\right]<0 .
\end{gathered}
$$

It can be found that the stability condition (23) is also satisfied. Since conditions (21-23) are all satisfied, it can be concluded that the discrete T-S fuzzy model (46) is asymptotically stable by applying the optimal filter gains $\boldsymbol{K}_{i}$ of (53) and observed-stated feedback gains $\boldsymbol{G}_{i}$ of (54).

In the simulation, the initial states are given as $\left[\begin{array}{ll}x_{1}(0) & x_{2}(0)\end{array}\right]^{T}=\left[\begin{array}{ll}-0.9 & 0.9\end{array}\right]^{T}$. Figure 2 and Figure 3 show

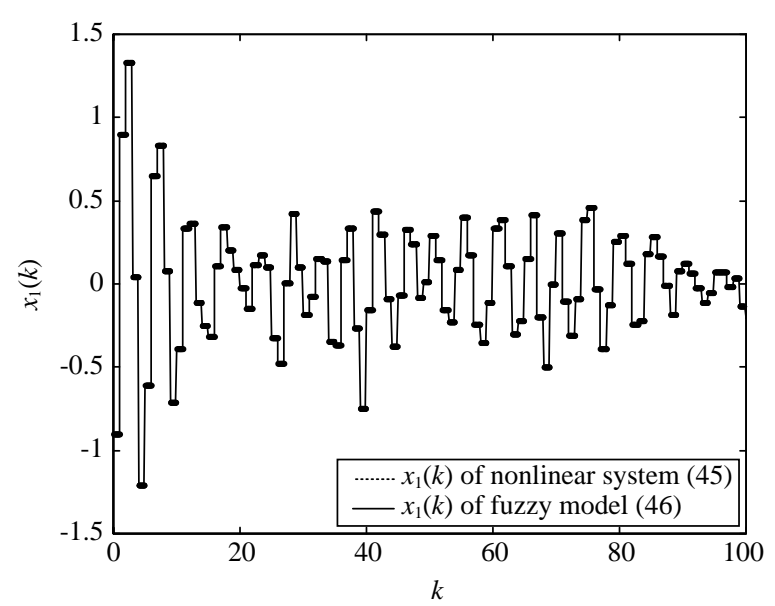

Fig. 2. The responses of $x_{1}(k)$ for controlled nonlinear system (45) and fuzzy model (46).

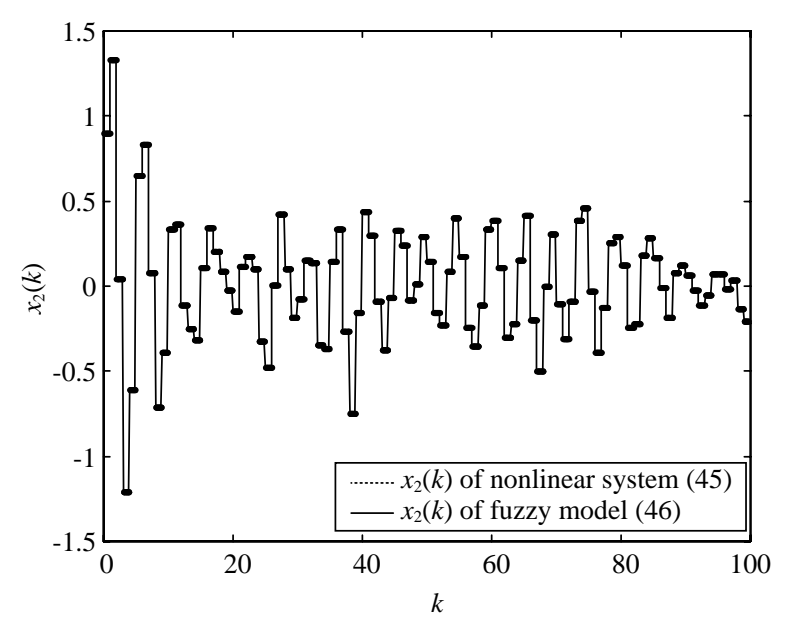

Fig. 3. The responses of $x_{2}(k)$ for controlled nonlinear system (45) and fuzzy model (46). 
the state responses of $x(k)$ of controlled nonlinear system (45) and T-S fuzzy model (46). Figure 4 and Figure 5 show the responses of true states $x(k)$ and the estimated state $\hat{x}(k)$ for the controlled T-S fuzzy model (46). From these simulation results, the state variances of closed-loop nonlinear system (45) are calculated as follows:

$$
\operatorname{var}\left(x_{1}(k)\right)=0.1287 \text { and } \operatorname{var}\left(x_{2}(k)\right)=0.1206
$$

where var $\left(x_{\ell}(k)\right)$ denotes the variance of system state $x_{\ell}(k), \ell=1,2$. It can be found that the closed-loop stochastic system is stable and the variance constraints (47) are achieved.

\section{CONCLUSIONS}

This paper considered the synthesis of nonlinear

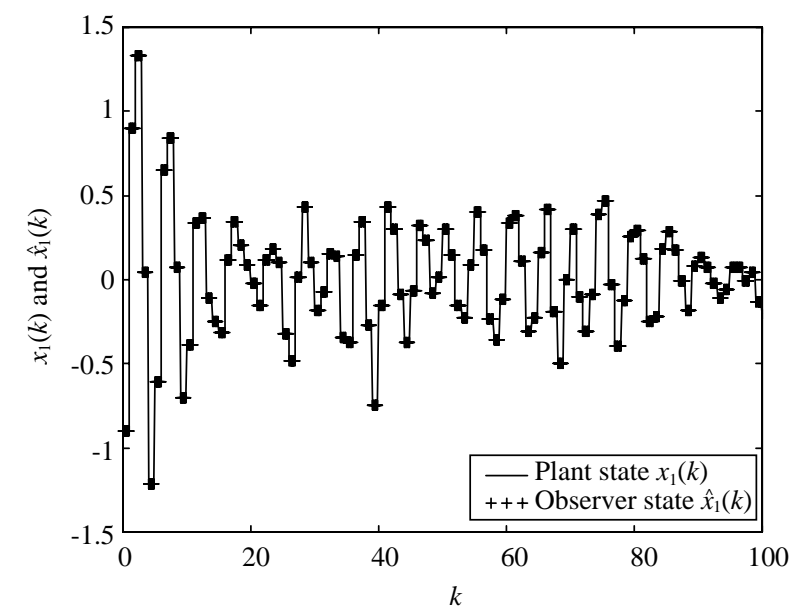

Fig. 4. The responses of true state $x_{1}(k)$ and estimated state $\hat{x}_{1}(k)$ for controlled fuzzy model (46).

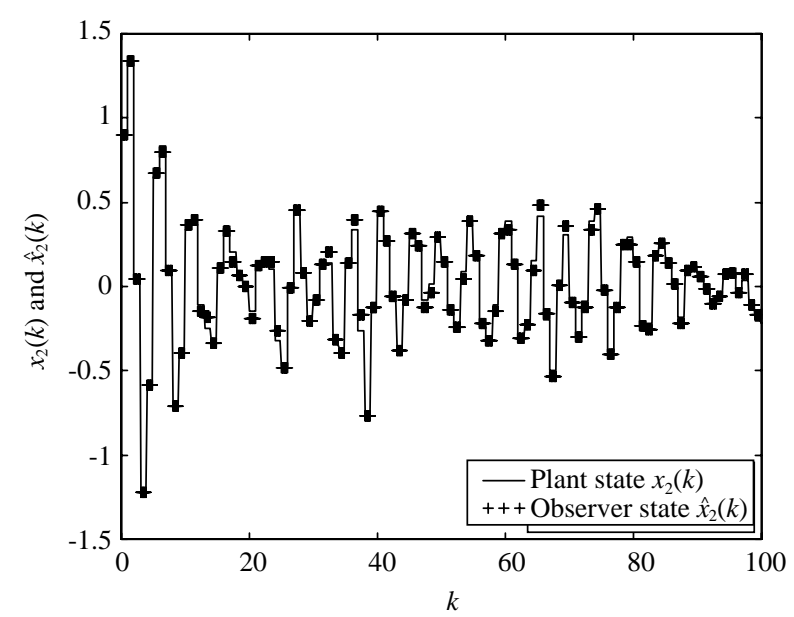

Fig. 5. The responses of true state $x_{2}(k)$ and estimated state $\hat{x}_{2}(k)$ for controlled fuzzy model (46). stochastic control systems, whose state variables cannot be completely measured. The nonlinear systems are modeled by the T-S type fuzzy models in this paper. The optimal filtering control technique has been used to design the observers for the T-S type fuzzy stochastic control systems. Applying these optimal observers, this paper first introduced the conditions for the existence of observed-state feedback gains. To carry on, the theory of generalized inverse was used to solve the observedstate feedback gains for the T-S fuzzy controllers. Based on the observed fuzzy control technique, the present approach allows the designers to assign the common state covariance matrix for achieving the state variance constraints.

\section{ACKNOWLEDGEMENTS}

The authors wish to express their sincere gratitude to three anonymous reviewers who gave them some constructive comments, criticisms and suggestions. This work was supported by the National Science Council of the Republic of China, under contract NSC93-2218-E019-002.

\section{REFERENCES}

1. Ben-Israel, A. and Greville, T.N.E., Generalized Inverse: Theory and Application, John Wiley \& Sons, New York (1974).

2. Bondaros, Y.G. and Konstantinov, V.M., "Estimation of the State of Linear Systems with Additive and Multiplicative Noise," Automatika Telemekhanika, Vol. 5, pp. 34-43 (1976).

3. Boyd, S., Ghaoui, L.E., Feron, E., and Balakrishnan, V., Linear Matrix Inequalities in Systems and Control Theory, SIAM, Philadelphia, PA (1994).

4. Chang, K.Y. and Chang, W.J., "Variable Structure Controller Design with $\mathrm{H}_{\infty}$ Norm and Variance Constraints for Stochastic Model Reference Systems," IEE Proceedings, Part D, Control Theory Applications, Vol. 146, No. 6, pp. 511-516 (1999).

5. Chang, K.Y., Wang, W.J., and Chang, W.J., "Covariance Control for Stochastic Multivariable Systems with Hysteresis Nonlinearity," International Journal of System Science, Vol. 28, No. 7, pp. 731-736 (1997).

6. Chang, W.J. and Chung, H.Y., "A Covariance Controller Design Incorporating Optimal Estimation for Nonlinear Stochastic System," ASME, Journal of Dynamic Systems, Measurement and Control, Vol. 118, No. 2, pp. 346-349 (1996).

7. Chang, W.J. and Shing, C.C., "Discrete Fuzzy Controller Design for Achieving Common State Covariance Assignment," ASME, Journal of Dynamic Systems, Measurement and Control, Vol. 126, No. 3, pp. 627-632 
(2004)

8. Chang, W.J. and Wu, S.M., "Covariance Control for Fuzzy-Based Nonlinear Stochastic Systems," International Journal of Fuzzy Systems, Vol. 5, No. 4, pp. 221228 (2003).

9. Chang, W.J., Sun, C.C., and Fuh, C.C., "Discrete Output Fuzzy Controller Design for Achieving Common Controllability Gramian," Asian Journal of Control, Vol. 2, No. 4, pp. 284-289 (2000).

10. Chung, H.Y. and Chang, W.J., "Constrained Variance Design for Bilinear Stochastic Continuous Systems," IEE Proceeding, Part D, Control Theory Applications, Vol. 138, No. 2, pp. 145-150 (1991).

11. Chung, H.Y. and Chang, W.J., "Covariance Control with Variance Constraints for Continuous Perturbed Stochastic Systems," Systems \& Control Letters, Vol. 19, No. 5, pp. 413-417 (1992).

12. Chung, H.Y. and Chang, W.J., "Constrained Variance Control with State Feedback for Bilinear Stochastic Discrete Systems," Journal of the Chinese Institute of Engineers, Vol. 16, No. 4, pp. 463-469 (1993).

13. Chung, H.Y. and Chang, W.J., "Extension of the Covariance Control Principle to Nonlinear Stochastic Systems," IEE Proceeding, Part D, Control Theory and Applications, Vol. 141, No. 2, pp. $93-98$ (1994).

14. Collins, Jr., E.G., "Covariance Control of Discrete System: Development and Applications," Ph. D Dissertation, Purdue University, West Lafayette, IN, USA (1987).

15. Collins, Jr., E.G. and Skelton, R.E., "A Theory of State Covariance Assignment for Discrete Systems," IEEE Transactions on Automatic Control, Vol. 32, No. 1, pp. 35-41 (1987).

16. Hotz, A.F. and Skelton, R.E., "Controller Design for Robust Stability and Performance," Proceedings of American Control Conference, Seattle, pp. 1813-1817 (1986).

17. Hotz, A.F. and Skelton, R.E., "A Covariance Control Theory," International Journal of Control, Vol. 46, No. 1 pp. 13-32 (1987).

18. Kwakernaad, H. and Sivan, R., Linear Optimal Control Systems, John Wiley \& Sons, New York (1972).

19. Mclane, P.J., "Optimal Linear Filtering for Linear Systems with State-Dependent Noise," International Journal of Control, Vol. 10, No. 1, pp. 41-51 (1969).

20. Pakshin, P.V., "State Estimation and Control Synthesis for Discrete Linear Systems with Additive and Multiplicative Noise," Automatic Remote Control, Vol. 4, pp. 526-535 (1978).

21. Phillis, Y.A., "Controller Design of Systems with Multiplicative Noise," IEEE Transactions on Automatic Control, Vol. AC-30, No. 10, pp. 1017-1019 (1985).

22. Phillis, Y.A., "Estimation and Control of Systems with Unknown Covariance and Multiplicative Noise," IEEE Transactions on Automatic Control, Vol. AC-34, No.
10, pp. 1075-1078 (1989).

23. Sreeram, V., Liu, W.Q., and Diab, M., "Theory of State Covariance Assignment for Linear Single-Input System," IEE Proceeding, Part D, Control Theory Applications, Vol. 143, No. 3, pp. 289-295 (1996).

24. Tanaka, K. and Sugeno, M., "Stability Analysis and Design of Fuzzy Control Systems," Fuzzy Set and Systems, Vol. 45, No. 2, pp. 135-156 (1992).

25. Tanaka, K. and Wang, H.O., Fuzzy Control System Design and Analysis - A Linear Matrix Inequality Approach, John Wiley \& Sons, New York (2001).

26. Tanaka, K., Ikeda, T., and Wang, H.O., "A Unified Approach to Controlling Chaos via An LMI-Based Fuzzy Control System Design," IEEE Transactions on Circuits and Systems I: Fundamental Theory and Applications, Vol. 45, No. 10, pp. 1021-1040 (1998).

27. Wang, H.O., Tanaka, K., and Griffin, M.F., "An Approach to Fuzzy Control of Nonlinear Systems: Stability and Design Issues," IEEE Transactions on Fuzzy Systems, Vol. 4, No. 1, pp. 14-23 (1996).

\section{APPENDIX}

Lemma A1 [1, 14]

Every matrix $\boldsymbol{J} \in \Re^{n \times n}$ of rank $m_{r}$ may be expressed as

$$
J=\Psi S
$$

where $\Psi$ is symmetric and positive semi-definite (i.e., $\Psi=\Psi^{T} \geq 0$ ) and $\boldsymbol{S}$ is orthonormal (i.e., $\boldsymbol{S} \boldsymbol{S}^{T}=\boldsymbol{I}$ ). $\Psi$ is always unique. If $m_{r}=n$, then $\boldsymbol{S}$ is unique. If $m_{r}<n$, then $S$ is nonunique.

\section{Lemma A2 [14]}

Express $\boldsymbol{J}$ using the singular value decomposition as $\boldsymbol{J}=\boldsymbol{Q} \Sigma \boldsymbol{T}^{T}$, where $\boldsymbol{Q}$ and $\boldsymbol{T}$ are orthonormal, $\Sigma=$ diag $\left(\sigma_{1}, \ldots, \sigma_{n}\right)$, and $\sigma_{1} \geq \sigma_{2} \geq \ldots \geq \sigma_{m_{r}}>0=\sigma_{m_{r}+1}=\ldots=\sigma_{n}$. Also, define

$$
\begin{aligned}
& \tilde{\boldsymbol{S}}=\left\{\boldsymbol{S}: \boldsymbol{S}=\boldsymbol{Q}\left[\begin{array}{cc}
\boldsymbol{I}_{r} & 0 \\
0 & \boldsymbol{U}_{0}
\end{array}\right] \boldsymbol{T}^{T}, \boldsymbol{U}_{0} \in \mathfrak{\Re}^{\left(n-m_{r}\right) \times\left(n-m_{r}\right)}\right. \\
& { }^{\text {r) }} \text { is arbitrarily orthonormal }
\end{aligned}
$$

Then, $\boldsymbol{J}=\boldsymbol{\Psi} \boldsymbol{S}$ as discussed in Lemma A1, if and only if $\boldsymbol{S} \in \tilde{\boldsymbol{S}}$ and $\boldsymbol{\Psi}=(\boldsymbol{J} J)^{\frac{1}{2}}=\boldsymbol{Q} \boldsymbol{\Sigma} \boldsymbol{Q}^{T}$. 